\title{
An agent-based model for estimating emissions reduction equivalence among leak detection and repair programs
}

Thomas A. Fox ${ }^{1 \dagger}$, Mozhou Gao ${ }^{2}$, Thomas E. Barchyn ${ }^{3}$, Yorwearth L. Jamin ${ }^{4 \ddagger}$, Chris H. Hugenholtz ${ }^{5}$

${ }^{\dagger}$ Centre for Smart Emissions Sensing Technologies, University of Calgary, 2500 University Drive NW, Calgary, AB, T2N 1N4, Canada

$\$$ Clearstone Engineering Ltd., 9006 Ave SW \#700, Calgary, AB, T2P 3K2, Canada

1 Correspondence to: thomas.fox@ucalgary.ca

2 mozhou.gao@ucalgary.ca

3 tbarchyn@ucalgary.ca

4 yori.jamin@clearstone.ca

5 chhugenh@ucalgary.ca

This is a preprint (not peer reviewed) submitted to EarthArXiv. This preprint has been submitted to the Journal of Cleaner Production and is currently in peer review. 


\begin{abstract}
Alternative leak detection and repair (alt-LDAR) programs are being introduced by regulators in North America to provide flexibility in how oil and gas producers manage their fugitive methane emissions. However, emissions reduction equivalence must be established between a proposed program and a regulatory standard. We present LDAR-Sim, an open-source, agent-based numerical model for estimating equivalence among LDAR programs and exploring specific LDAR scenarios. Novel advancements include the ability to: (1) set facility-specific LDAR requirements and deployment constraints, (2) simultaneously deploy multiple technologies, each with multiple intelligent agents, (3) integrate screening and close-range methods in a collaborative work practice, (4) consider unique environmental limitations of different technologies, (5) evaluate the impact of limited equipment and labour, and (6) explore the impact of legally vented emissions on screening technologies. We examine several alt-LDAR scenarios using real assets and discuss model confidence and sensitivity to inputs. We show that equivalence determinations depend on explicit definition of reference standards, including weather and labour availability. Screening method performance is vulnerable to the confounding presence of vented emissions and to criteria that trigger follow-up surveys. Relative mitigation of programs is highly sensitive to leak production and null repair rates, two elusive parameters used in previous studies.
\end{abstract}




\section{Introduction}

Oil and gas systems are the largest source of anthropogenic methane emissions in Canada and the US. ${ }^{1,2}$ In the upstream O\&G sector, methane can be emitted by design (i.e. intentional vented emissions) or as accidental leaks (i.e. unintentional fugitive emissions). To address fugitive emissions, regulators often require producers to implement leak detection and repair (LDAR) programs. ${ }^{3,4}$ Most LDAR programs consist of periodic surveys using handheld instruments such as optical gas imaging (OGI) cameras or organic vapour analyzers. These 'conventional' surveys are conducted at the component scale using close-range methods with high sensitivity.

Alternative approaches to LDAR (alt-LDAR) may exist. ${ }^{5,6} \mathrm{We}$ define alt-LDAR as any deviation from a prescribed regulatory LDAR program (reg-LDAR). Over the past decade, a variety of technologies, methods, and program designs have emerged that may contribute to improving the cost- and mitigationeffectiveness of LDAR. In response, various regulatory agencies in the US and Canada have moved to recognize alt-LDAR as a potentially viable path to compliance. ${ }^{3,7}$ Regulators often require evidence that proposed alt-LDAR programs achieve equivalent mitigation to the reg-LDAR standard. A combination of controlled testing, simulation modeling, and field trials has been recommended. ${ }^{8}$

Simulation modeling provides several advantages as a method of understanding the emissions reductions potential of an LDAR program. First, simulations allow a long-term estimation of emissions dynamics. Second, direct testing of LDAR tools on real leaks is slow, expensive, and possibly unfair as a representative distribution of emission rates is difficult to acquire. ${ }^{9}$ Third, empirically measuring total mitigation progress is challenging due to the high uncertainties typical of most quantification methods. ${ }^{10}$ The first LDAR simulation tool was the Fugitive Emissions Abatement Simulation Testbed (FEAST). ${ }^{11}$ FEAST generates a virtual leak field in which leaks arise and are removed stochastically at the component scale following a Markov process. Detection modules are then applied to the leak field. When a survey is conducted at a component, detection is evaluated by simulating a Gaussian plume and considering the physical properties of the sensor. If detected, leaks are repaired and the component's Markov state 
switches to not emitting. Mitigation is calculated as the difference between time-integrated emissions under the LDAR program and a 'null' scenario.

The original version of FEAST is a valuable tool but has drawbacks that may limit its broader utility. First, the success of an LDAR program depends not only upon the theoretical detection capabilities of a method, but also upon practical considerations that vary by producer, facility, environment, and policy context. For example, an aircraft-based remote sensing system may perform poorly in high-latitude winters due to snow cover, cloud cover, and low solar irradiance. An alt-LDAR program that commits to monthly surveys using this system may only be successful in specific geographical or seasonal contexts. If only a handful of days are suitable for deployment, labour and equipment availability may become limited, especially if only one aircraft or crew exists. Second, LDAR simulation tools should provide producers with an opportunity to explore and optimize across a range of possible programs. For example, a producer may propose a program that simultaneously integrates aircraft, trucks, and OGIs with facilityspecific survey schedules and frequencies, and custom repair protocols. Important interaction effects may emerge from concurrent deployments that could influence the equivalence determination.

To meet these needs, we present LDAR-Sim, an open-source agent-based framework that permits the simultaneous deployment of multiple methods, each with multiple agents (crews), while allowing for the definition of custom LDAR programs. We first present a detailed description of LDAR-Sim, then demonstrate the model with a case study using real assets in Alberta, Canada. We conclude with a comprehensive sensitivity analysis (SA) and discussion of modeling assumptions and shortcomings, and their implications for generating equivalence determinations.

\section{Model description}

LDAR-Sim uses the initialize-update-finalize Basic Model Interface of the Community Surface Dynamics Modeling System. ${ }^{12}$ Each program consists of a set of assets, one or more leak detection methods, and a set of rules governing deployment and repair. Assets are combined with empirical inputs to initialize a 
leak field. A dynamic simulation with daily timesteps and minute-scale temporal resolution is then used to evaluate the program (Figure 1). Leaks arise stochastically and are detected probabilistically according to method-specific empirical detection data. Aggregate-scale detections (i.e. by screening methods) can produce flags, which trigger follow-up by close-range methods. Leaks detected at close-range are tagged and enter a repair queue. Tagged leaks are removed from the simulation following user-defined reporting delays and repair intervals. Parameterization, selection of empirical inputs, and sensitivity analyses (SA) should be done on a case-by-case basis; no defaults exist.

\subsection{Program definition}

A primary motivation in developing LDAR-Sim is to enable the precise definition of custom LDAR programs. Defining a program requires identifying the detection method(s), the asset base, and deployment protocols. In practice, each method module should be designed in accordance with the technology and work practice proposed by the solution provider. Ideally, a set of relevant performance metrics are developed through controlled testing. We provide a set of general method templates, including OGI, a mobile ground laboratory (MGL), and an aircraft system. Each method can be parameterized according to relevant criteria, which commonly include detection performance, labour capacity, minimum survey intervals, environmental constraints, reporting delays, and follow-up criteria for screening methods. These templates can also be used to develop modules for other methods.

The quality of the parameterization depends on the empirical data available. For example, detection performance can be a static minimum detection limit or a function of leak size and environmental conditions. Labour capacity is defined by the number of unique agents (crews) that can be deployed, the duration of a workday (including daylight constraints), the time to complete each facility, and time spent between facilities, which combines transit, permitting, breaks, and complications. For reg-LDAR, minimum survey intervals reflect policy requirements designed to spread out surveys. For alt-LDAR, these intervals represent clearly-defined work practices within a proposed program. Reporting delays represent the time interval from detection to actionable information. Delays may be trivial for some 
methods, but functionality has been included for methods that are slow to process and deliver actionable information.

Environmental conditions can be incorporated in two ways. First, operational envelopes are defined, preventing deployment if conditions are unsuitable (a binary determination as defined by each method's work practice). Second, detection performance within an envelope can be configured as a function of the environment through a custom model relating environment to performance. As environmental conditions vary in space and time, so should the performance of LDAR methods. To account for local environmental context, LDAR-Sim uses gridded ERA-Interim reanalysis data. These data have global daily coverage with $\sim 80 \mathrm{~km}$ spatial resolution from the European Centre for Medium-Range Weather Forecasts. See Dee et al. for a comprehensive description of these data. ${ }^{13}$ At a minimum, LDAR-Sim requires temperature, wind speed, and precipitation as inputs, as these are known to impact the performance of OGIs, ${ }^{14}$ which are typically used as the reference target in equivalence. ${ }^{3,4}$ However, the reanalysis contains a detailed description of the weather, including wind direction (for downwind plume intersection methods), snow depth and cloud cover (for remote sensing methods), and solar irradiance, which can be incorporated as needed.

Facilities in the LDAR program (henceforth 'sites') are provided to LDAR-Sim as geographic coordinates to establish local environmental context and topology. A single site could be used, a site list comprised of a company's assets, or all sites in a basin or jurisdiction. Custom survey frequencies can be assigned to each site for each method used in a program. These can be uniform for simple scenarios or based on facility type or risk factors such as production, age, or site-specific historical LDAR data. Survey frequencies can also be set according to accessibility. For example, if a site is not accessible by MGL, it can be designated an OGI site. The time required to survey each site can also be specified by method. 


\subsection{Initialization}

To start the simulation, methods and site emissions are initialized. In LDAR-Sim the user can simultaneously deploy multiple methods, each with one or more independent crews. To achieve this structure, each method has a 'company' that manages daily work requirements by deploying crews. This construct also enables optimization of crew management. Each company in the program is initialized by building a deployment day matrix defining whether each site is available on each day of the simulation according to the environmental constraints set during program definition. This results in $n_{m}$ matrices of dimensions $n_{s}$ and $t$, where $n_{m}$ is the number of methods in the program, $n_{s}$ is the number of sites, and $t$ is simulation timesteps in days. To register a deployment day, all environmental conditions at a site must satisfy the user-defined operational envelopes for that method.

Site emissions are initialized as fugitive and vented emissions. For each site, the number of initial leaks is randomly drawn from an empirical distribution of leak counts, and each leak is assigned an emission rate from an empirical leak-size distribution. Bias can arise if count and rate distributions are not acquired using the same detection method. For example, if counts are acquired from a study using Method 21 instruments and rates from a study using OGIs, aggregate emissions will be overestimated due to the lower sensitivity of OGIs. Ideally, both count and rate data should come from the same study and be representative of the region or assets of interest. If local data are limited or unavailable, inputs should be as representative as possible, and sensitivity should be tested.

Most LDAR programs target only fugitive emissions, but allowable vented emissions matter because methods that measure site-level emissions are often unable to distinguish between the two. Previous studies and inventories have commonly attributed $>50 \%$ of site-level emissions to vents, ${ }^{15,16}$ so it is important to consider the potentially confounding effects of these sources. To date, 'bottom-up' component-level measurements have been difficult to reconcile with contemporaneous 'top-down' sitelevel measurements ${ }^{17}$. Possible explanations for the large discrepancy include (1) inability to measure certain sources due to access restrictions (e.g. tank vents) or instrumentation limitations (e.g. compressor 
exhaust lacks quality measurement methods), and (2) increased chance that top-down methods capture episodic events such as blowdowns and liquid unloadings ${ }^{16,18}$. Rather than attempt to estimate site-level vented emissions $\left(V_{i}\right)$ from component-level measurements, LDAR-Sim takes an empirical set of sitelevel measurements and bootstraps the count $(C)$, leak $(L)$, and site-total $(T)$ empirical distributions to estimate site-level vented emissions:

$$
V_{i}=T_{i}-\sum_{j=1}^{C_{i}} L_{j}
$$

This procedure generates a distribution of $i$ (typically 1000) site-level vented emission estimates.

Whereas leaks are assigned to sites and remain at the same site, vents are assigned stochastically during each survey to simulate the episodic and dynamic nature of vented emissions ${ }^{19,20}$.

\subsection{Updating}

After initialization, LDAR-Sim evaluates the program at daily timesteps. Each day can be split into three parts: adding new leaks, finding leaks, and repairing leaks.

\subsubsection{Adding new leaks}

New leaks $\left(L_{n}\right)$ are generated using a site-level empirical leak production rate (LPR; leaks $\cdot$ site $^{-1} \cdot$ day $\left.^{-1}\right)$ that is independent of the number of leaks already present on site:

$$
L_{n} \sim \operatorname{Binomial}(1, L P R)
$$

In theory, each site can have $t+L_{i}$ leaks, where $L_{i}$ is the initialized leak count. This is unrealistic and is made impossible in FEAST, which limits the number of leaks at a site to a finite component count. However, leak removal processes described in the following section prevent unrealistic accumulation of leaks, and we find that specifying an upper limit is unnecessary. In LDAR-Sim only one new leak can be added to a site per day. However, for a typical LPR of 0.006 , there is only a $3.6 \times 10^{-5}$ chance that two 
new leaks will arise at a site on the same day (approximately once every 65 years); we do not consider this an important concern.

\subsubsection{Finding leaks}

In LDAR-Sim, we distinguish between close-range methods that are able to confirm and diagnose leaks at the component scale, and screening methods that measure at larger spatial scales. Leaks must be detected at the component scale before they can be tagged for repair and enter the repair queue. This can happen in two ways: by operators during routine visits to their sites or with close-range LDAR (e.g. OGI). Methods measuring at the equipment or facility scale (e.g. MGLs, aircraft, satellites, and most drones) must flag sites for follow-up by a close-range method as they are generally unable to discern individual sources or distinguish between vented and fugitive emissions. Operator detection represents periodic inspection, maintenance, and repair outside of an LDAR program and is governed by a null repair rate (NRR). An overview is presented in S1 with further discussion in Sections 2.4.1 and 4 below.

When an LDAR program is configured, each company in the program is instructed to work each day. Companies then deploy each of their crews and leak detection occurs in accordance with the method's work practice, user-defined performance metrics, and program requirements. When a crew is deployed, workday duration is first calculated as the shorter of either (1) the number of daylight hours, or (2) the user-defined maximum workday duration for that method. Daylight hours are calculated using the ephem package in python, which returns civil twilight sunrise and sunset times for a given date, latitude, and longitude ${ }^{21}$. The crew then 'chooses' and 'visits' sites until the workday ends.

Crews attempt to visit sites that have gone the longest without LDAR. A series of checks ensures that (1) the minimum interval has passed between surveys, (2) the site is not yet in compliance for the year (to avoid conducting too many surveys), and (3) environmental conditions fall within the operational envelope. If any of these conditions are not met, the crew considers another site. If no suitable sites are identified, the workday ends. If a site is identified, the crew visits it and each leak is subject to the crew's detection module. Detected leaks are tagged for repair. Once all leaks have been attempted, the time of 
day for the crew advances by the sum of: (1) the site-specific survey time for the crew's method, and (2) an estimate of the time required to reach the next site.

Screening method templates are similarly configured, but flag entire sites for follow-up by a close-range method. When a site is surveyed, the method's detection module is applied to the cumulative site emissions $\left(Q_{\text {site }}\right)$, which consists of all leaks and a vented emissions estimate $(V)$ drawn from the empirical distribution of vents:

$$
Q_{\text {site }}=V+\sum_{l=1}^{n_{\text {leaks }}} Q_{l}
$$

If a site is detected it can be flagged, but only if $Q_{\text {site }}$ exceeds an optional user-defined follow-up threshold. The purpose of the threshold is to prevent highly sensitive screening methods from sending follow-up crews to each site they screen. A 'follow-up ratio' may also be specified, which flags sites based on relative emissions. For example, a follow-up ratio of 0.2 will flag the top $20 \%$ of surveyed sites.

\subsubsection{Repairing leaks}

Leaks are repaired following optional reporting and repair delays. All methods have a reporting delay separating data collection from delivery of actionable information. For screening, two reporting delays exist in the flag-tag-repair workflow as a liaising follow-up crew is required. Once tags are reported, a repair delay must pass. The repair delay can be a single value or an empirical distribution. The true distribution is unknown but may be skewed or bimodal as some leaks can be repaired immediately (e.g. tightening a valve) while others require an equipment shutdown.

\subsection{Finalization}

When all simulation days are complete, LDAR-Sim outputs a series of data files, maps, and figures. Data are output by site, leak, and day to enable detailed and transparent evaluation of results (Table S1). The proportion of sites available is a global measure of suitability reflecting the daily proportion of all sites that fall within each method's environmental constraints. Although cost is not presently treated 
thoroughly in LDAR-Sim, users are encouraged to build their own costing modules with real data. Our placeholder cost variable is a crude estimate of daily company costs and is charged for each crew deployed.

When multiple methods are deployed, it is important to track different kinds of detection redundancy that may arise. Redundant tags are tagged leaks that are detected a second time by an operator or close-range method. These tags are not counted, as the leak is already waiting repair. Three types of redundant flags exist and are described in Figure S1. Tracking redundancy is important to ensure that multiple companies or crews are not taking credit for emissions reductions resulting from the repair of the same leak.

\subsubsection{Measuring equivalence}

In FEAST, mitigation was estimated by subtracting emissions under an LDAR program from a null scenario - 'business as usual' in the absence of LDAR. ${ }^{14}$ The null scenario balances LPR and NRR in the absence of LDAR to achieve steady-state emissions centered on the initialized state of the leak field.

We find the notion of a null scenario problematic for several reasons. First, the 'pre-LDAR' baseline is elusive, and uncertainty in its estimate propagates to mitigation. Today, many companies have some kind of leak management program already in place. In Canada, a shift towards more formalized LDAR occurred in 2007 when best management practices were published by an industry group. ${ }^{22}$ Second, fugitive emissions are unlikely to be in equilibrium. Management practices, new technology, and pressure on producers to reduce emissions may result in a downward trend in fugitive emissions. While both possibilities are speculative, the burden of proof should be on equilibrium if this assumption is required. Third, the null baseline is maintained by LPR and NRR, two poorly understood parameters. Both LPR and NRR have significant challenges:

- LPR has never been estimated independently of NRR; in deriving LPR, it is assumed that NRR and LDAR are inconsequential. This is inconsistent with assumptions made during simulation, 
where NRR is assigned a value approximately equal to LPR. If estimating an LPR that already encodes null repair, NRR should not need to be applied during simulation.

- $\quad$ LPR is most easily estimated from data acquired during LDAR surveys, compromising the 'preLDAR' steady state assumption.

- While attempts have been made to estimate LPR, NRR has neither been measured nor empirically inferred.

- In FEAST, NRR is a static value defined using LPR. The same NRR is used for all leaks, regardless of emissions rate. However, large leaks may be more easily detected by human senses, and if discovered, are of greater safety and financial concern. If removal rate is a function of leak size, production of larger leaks must increase to maintain the relative proportion of large leaks.

- NRR likely depends on company culture and individual operator practice. In FEAST, a higher LPR results in a higher NRR. However, if company culture matters, LPR and NRR may be inversely related.

Given these assumptions, it is conceivable that the best guesses for LPR and NRR are wrong, perhaps by a large margin. We therefore avoid the concept of a null scenario and compare programs directly based on emissions instead of mitigation. This can be formalized: Let equivalence between two programs $\left(P_{1}\right.$ and $\left.P_{2}\right)$ be defined as equal mitigation $\left(M_{1}=M_{2}\right)$. Assume that $M=\operatorname{Null}_{E}-P_{E}$, or emissions from the null scenario minus program emissions. If the null scenario is the same for both programs, equivalence can be defined as $\mathrm{Null}_{E}-P_{E 1}=\mathrm{Null}_{E}-P_{E 2}$, where $\mathrm{Null}_{E}$ cancels out.

\section{Case study}

To demonstrate LDAR-Sim, we present a case study of two alt-LDAR programs deployed using real assets in Alberta, Canada. Both programs are implemented over a 7-year period (2011-2018; using historical weather data) alongside the OGI-based regulatory standard required by Environment and Climate Change Canada (ECCC) ${ }^{4}$. Briefly, ECCC requires triannual surveys on most upstream 
production facilities, with the exception of single wellheads with no associated equipment (i.e. connected directly to a gathering pipeline). Most leaks must be repaired within 30 days, unless a major shutdown is required. We demonstrate these programs for a producer with 1169 Alberta facilities requiring LDAR. To maintain anonymity of the producer, random noise $\mathcal{N}(0,0.1)$ was added to the $\mathrm{x}$ and y coordinates of each site and no identifying information is reported. This has negligible effect on the conditions polled from weather data. The sites are broadly representative of Alberta's diverse geography and producing regions, spanning approximately $9.6^{\circ}$ of longitude and $8.7^{\circ}$ of latitude. For each program we conduct 25 simulations over 2557 daily timesteps and assume a standard repair delay of 14 days for all leaks. Input parameters are summarized in Table 1 and explained in S2.

Case study results are presented in Figure 2 and Table S2. In general, emissions in Figure 2 increase according to the LPR and decrease when LDAR is conducted. Each program configuration leads to distinct emissions trends (Figure 2). Only $\mathrm{P}_{1}$ achieves equivalence to the reference case. Although less sensitive (the OGI median detection limit is $\sim 10 \mathrm{~g} \cdot \mathrm{h}^{-1}$ ), $\mathrm{P}_{1}$ measures cumulative site-level emissions, including vents, and sends follow-up crews to $80 \%$ of detected sites. In this configuration, $\mathrm{P}_{2}$ is not equivalent to $P_{\text {ref }}$ as it is only visiting sites emitting above $5000 \mathrm{~g} \cdot \mathrm{h}^{-1}$. Small leaks are still eventually resolved, as follow-up crews conduct comprehensive surveys when tasked to a high-emitting site. Despite large differences in emissions (and therefore mitigation), each of the programs results in roughly the same number of tagged leaks (Table S2). This suggests that screening methods may be less efficient than closerange methods, because (1) the presence of vented emissions can influence follow-up decisions, and/or (2) higher detection limits require more leaks to build up before a follow-up is triggered.

Periodicity in emissions is driven by survey frequency, labour availability, and deployment constraints. The slope of emission reductions relates to the size of the workforce relative to the number of facilities to be surveyed. In FEAST, this slope is vertical, creating a 'saw tooth' emissions profile not observed in LDAR-Sim because agents typically require several weeks or months to complete each round of surveys. The absence of periodicity in $\mathrm{P}_{\mathrm{L}}$ and $\mathrm{P}_{1}$ suggests that agents are working continuously. The triannual 
fluctuations in $\mathrm{P}_{\text {ref }}$ suggest that crews survey all required sites before the next round of surveys is required. A balance must be found between having idle crews and a risk of falling out of compliance. Strong periodicity can be indicative of an oversupply of labour or of operational envelopes restricting surveys to seasonal clusters. The weather-restricted programs ( $\mathrm{P}_{\mathrm{W}}$ and $\left.\mathrm{P}_{\mathrm{W}, \mathrm{L}}\right)$ exhibit strong seasonal (wintertime) periodicity resulting in $\sim 50 \%$ higher fugitive emissions (Figure $2 \mathrm{C}$, Table S2). To better understand how weather affects deployment days, LDAR-Sim generates maps to explore the spatial distribution of suitable conditions for a given set of environmental constraints (Figure S2).

The variable performances of the OGI-based programs in Figure $2 \mathrm{C}$ illustrate the importance of defining the reference program for determining equivalence. Most jurisdictions require demonstration that altLDAR is equivalent to reg-LDAR but are not explicit about the quantity of emissions permitted or mitigated. When $\mathrm{P}_{2}$ is compared to $\mathrm{P}_{\text {ref }}$ it does not achieve equivalence. However, $\mathrm{P}_{2}$ is closer to $\mathrm{P}_{\mathrm{W}, \mathrm{L}}$ and could be deemed equivalent. To achieve defensible definitions for emissions reductions, regulators should be explicit in delineating permissible contexts or expected mitigation for equivalence demonstration.

\section{Model confidence}

Building confidence in LDAR models is challenging because (1) many of the parameters used to characterize leak fields, detection methods, and repair remain poorly constrained, (2) empirical inputs, and therefore results, vary in time and space, and (3) validation capacity is limited by the high cost of LDAR programs, the long periods over which they can be implemented, and the challenge of accurate emissions measurement. Although the value of these tools is heuristic, model confidence can be established by comparing similar models, conducting SA, and comparing against real LDAR programs.

The only model that can be used for comparison is FEAST, which has similar leak field properties but differs in most other aspects. Results from FEAST simulations of OGI-based LDAR programs are presented in two studies. ${ }^{11,14}$ Both use the same LPR as our case study, but all three studies use different empirical leak distributions. In the first, fugitive emissions fluctuate between $\sim 1-10 \mathrm{~kg} \cdot \mathrm{day}^{-1} \cdot \mathrm{site}^{-1}$ for a 
survey interval of 100 days. ${ }^{11}$ In the second, quarterly and annual surveys result in mean emissions rates of $\sim 13$ and $26 \mathrm{~kg} \cdot$ day $^{-1} \cdot$ site $^{-1}$, respectively. ${ }^{14}$ Results from LDAR-Sim's OGI demonstration fall within these two studies $\left(\sim 3-13 \mathrm{~kg} \cdot \mathrm{day}^{-1} \cdot \mathrm{site}^{-1}\right.$; Figure 2$)$, although neither model has been validated against field measurements.

For equivalence, accurately estimating emissions under an LDAR program may be less important than estimating the relative difference between two programs. To explore how different parameterizations could impact equivalence determinations, we compare an OGI-based program to a generic MGL screening program in an extensive SA (see S3 for methods).

Results show that leak production rate is the most sensitive input parameter (Figure 3), similar to past results. ${ }^{11}$ As LPR increases, the absolute difference in emissions between programs increases (Figure 4a), with the OGI program outperforming the screening program. However, relative emissions between programs appear independent of LPR $(S=-0.3$ for ratio and 1.7 for absolute difference; Figure 3$)$, suggesting that detection capacity of different programs may scale with total emissions, despite falling behind in absolute terms. Given that LPR remains an elusive parameter, we recommend comparing programs on a relative rather than absolute basis. If absolute differences in emissions are used, establishing equivalence may be easier for low-LPR companies or regions. Future research of repeat field surveys should feature robust record keeping of repairs and leak history (new, re-occurred, and not repaired) to improve LPR estimates.

Follow-up work practices and the presence of venting impact the performance of screening programs (Figure 3). Screening methods that measure site-level emissions are unable to distinguish between vented and fugitive sources. As vented emissions increase, follow-up decisions may preferentially target highventing sites at the expense of fugitive emissions. The impact of venting on relative performance is higher when LPR is low (Figure 4b), likely because fugitive emissions are lower and more likely to be overwhelmed by vented emissions, which are independent of LPR. The SA suggests that increasing follow-up ratios and decreasing thresholds can improve screening performance. For alt-LDAR programs 
to be appealing to producers, trade-offs between follow-up rules and deployment costs should be considered.

Results are sensitive to assumptions surrounding operator activity (Figure 3). As more leaks are repaired by operators, fewer remain for LDAR programs. Higher operator activity therefore results in smaller differences between programs, making demonstration of equivalence easier. The operator effect tends to increase with LPR, but mostly when comparing absolute differences in outputs. Given the evidence available, we advocate for disabling NRR in most situations, because (1) most empirical LPR estimates implicitly encode NRR, making additional NRR activity redundant; (2) no empirical NRR data exist and AVO detection metrics have not been established; (3) the assumption that system-wide fugitive emissions are in equilibrium lacks evidence; and (4) incorporating NRR obscures program comparison, increasing the threat of erroneously declaring two programs equivalent. Although LPR and NRR are nearly impossible to measure separately, they are relatively easy to measure together. Rather than attempt to estimate both LPR and NRR independently, we recommend modelers (1) reject the requirement of a steady-state and a null scenario and (2) work only with the empirical LPR that already accounts for NRR.

Several input variables are of seemingly low influence (Figure 3). For example, LDAR-Sim outputs do not appear to be sensitive to the addition or removal of super-emitters in any of the empirical input distributions. Others include operator bonus $\left(p_{\max }\right)$, detection limits for MGLs, and leak-size distributions. However, these inputs may still be important as the SA is designed to identify the most influential outputs. Targeted SAs are therefore necessary each time LDAR-Sim is used, as input variables may become sensitive when tested against a narrower range of other inputs.

Additional variables and functionality not considered in our case study or SA may be important, depending on program configuration. More flexibility in spatial resolution should be built into LDARSim, as some methods monitor at the equipment scale, providing more detailed information than facilityscale screening. Other possible improvements include quantification accuracy of screening methods, 
additional environmental variables to define operational envelopes of new methods, and repair rates. The modular structure of LDAR-Sim enables straightforward incorporation of new variables and functionality. At present, collective understanding of how to configure LDAR programs is nascent. Many of the inputs required to define LDAR programs are poorly constrained. Detection modules presented here are based on hypothetical performances and are not tied to specific work practices. To be used effectively, custom modules should be developed for each candidate method. These modules should reflect each method's unique set of performance metrics developed through controlled testing. Field data will improve understanding of these variables and identify others that may warrant inclusion. By means of open-source development, LDAR-Sim can evolve alongside this knowledge base, and may grow to be used in different ways by regulators, producers, consultants, solution providers, technology developers, and nonprofits. Future work will identify alt-LDAR programs that improve upon reg-LDAR, develop methods to optimize cost to mitigation ratios, validate model predictions through field trials, and improve integration of empirical inputs. 


\section{Figures and tables}
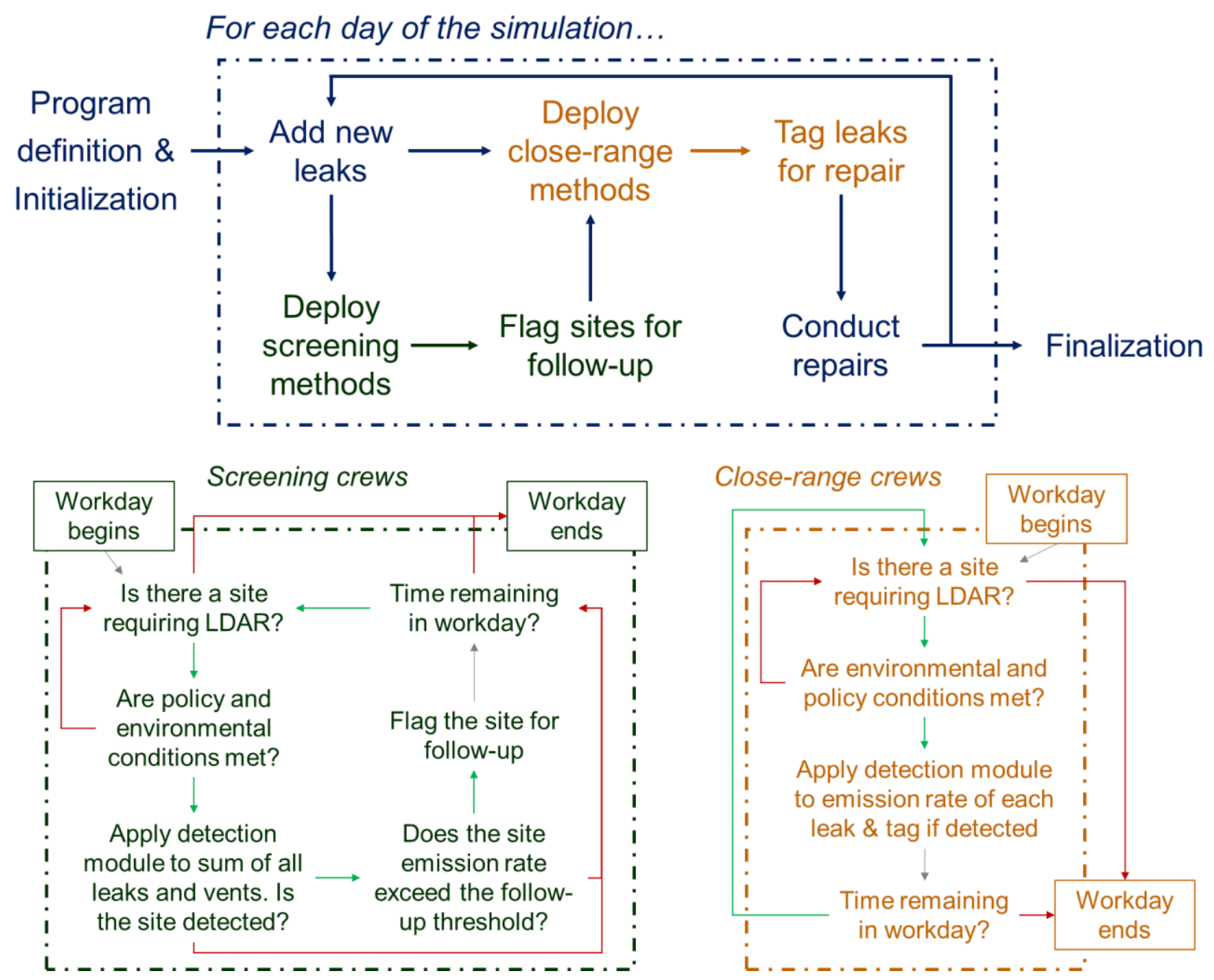

Figure 1. Overview of LDAR-Sim. Green text is used for screening methods and orange text for closerange methods. Red arrows represent 'no', green arrows are 'yes', and grey arrows are mandatory. 
Table 1. Parameters used for the case study. These programs are hypothetical and based on fabricated detection methods and survey requirements for the sake of demonstration.

\begin{tabular}{|c|c|c|c|c|c|c|c|}
\hline & $\mathrm{P}_{\text {Ref }}$ & $\mathrm{P}_{\mathrm{W}}$ & $\mathrm{P}_{\mathrm{L}}$ & $\mathrm{P}_{\mathrm{W}, \mathrm{L}}$ & $\mathrm{P}_{1} *$ & $\mathrm{P}_{2} *$ & $\dagger$ \\
\hline Method & OGI & OGI & OGI & OGI & MGL & Aircraft & OGI \\
\hline Number of crews & 3 & 3 & 1 & 1 & 1 & 1 & 2 \\
\hline Minimum temp $\left({ }^{\circ} \mathrm{C}\right)$ & -30 & 0 & -30 & 0 & -30 & -30 & -30 \\
\hline Maximum wind speed $\left(\mathrm{m} \cdot \mathrm{s}^{-1}\right)$ & 20 & 5 & 20 & 5 & 20 & 20 & 20 \\
\hline Maximum precipitation $(\mathrm{m})^{\ddagger}$ & 0.01 & 0.001 & 0.01 & 0.001 & 0.01 & 0.01 & 0.01 \\
\hline Minimum repeat interval (days) & 120 & 120 & 120 & 120 & 50 & 50 & - \\
\hline Maximum workday (hours) & 10 & 10 & 10 & 10 & 10 & 10 & 10 \\
\hline Reporting delay (days) & 2 & 2 & 2 & 2 & 2 & 2 & 2 \\
\hline Detection limit $\left(\mathrm{g} \cdot\right.$ hour $\left.^{-1}\right)$ & $\mathrm{a}$ & a & a & $\mathrm{a}$ & 100 & 5000 & $\mathrm{a}$ \\
\hline Time offsite (minutes $\cdot$ site $^{-1}$ ) & $\mathrm{b}$ & $\mathrm{b}$ & $\mathrm{b}$ & $\mathrm{b}$ & $\mathrm{b}$ & 10 & $\mathrm{~b}$ \\
\hline Required surveys per site & 3 & 3 & 3 & 3 & 4 & 6 & - \\
\hline Time per site (minutes) & 120 & 120 & 120 & 120 & 30 & 5 & 120 \\
\hline Follow-up threshold $\left(\mathrm{g} \cdot\right.$ hour $\left.^{-1}\right)$ & - & - & - & - & 100 & 5000 & - \\
\hline Follow-up ratio & - & - & - & - & 0.8 & 0.5 & - \\
\hline \multicolumn{8}{|c|}{ * Screening programs include two OGI follow-up crews } \\
\hline \multicolumn{8}{|c|}{$\dagger$ This column gives relevant follow-up parameters for screening methods. } \\
\hline \multicolumn{8}{|c|}{$\$$ Accumulated between 12:00 and 18:00 } \\
\hline \multicolumn{8}{|c|}{${ }^{a}$ Configured using Ravikumar et al (2018). See text for details. } \\
\hline \multicolumn{8}{|c|}{${ }^{\mathrm{b}}$ Drawn from an empirical distribution. See text for details. } \\
\hline
\end{tabular}




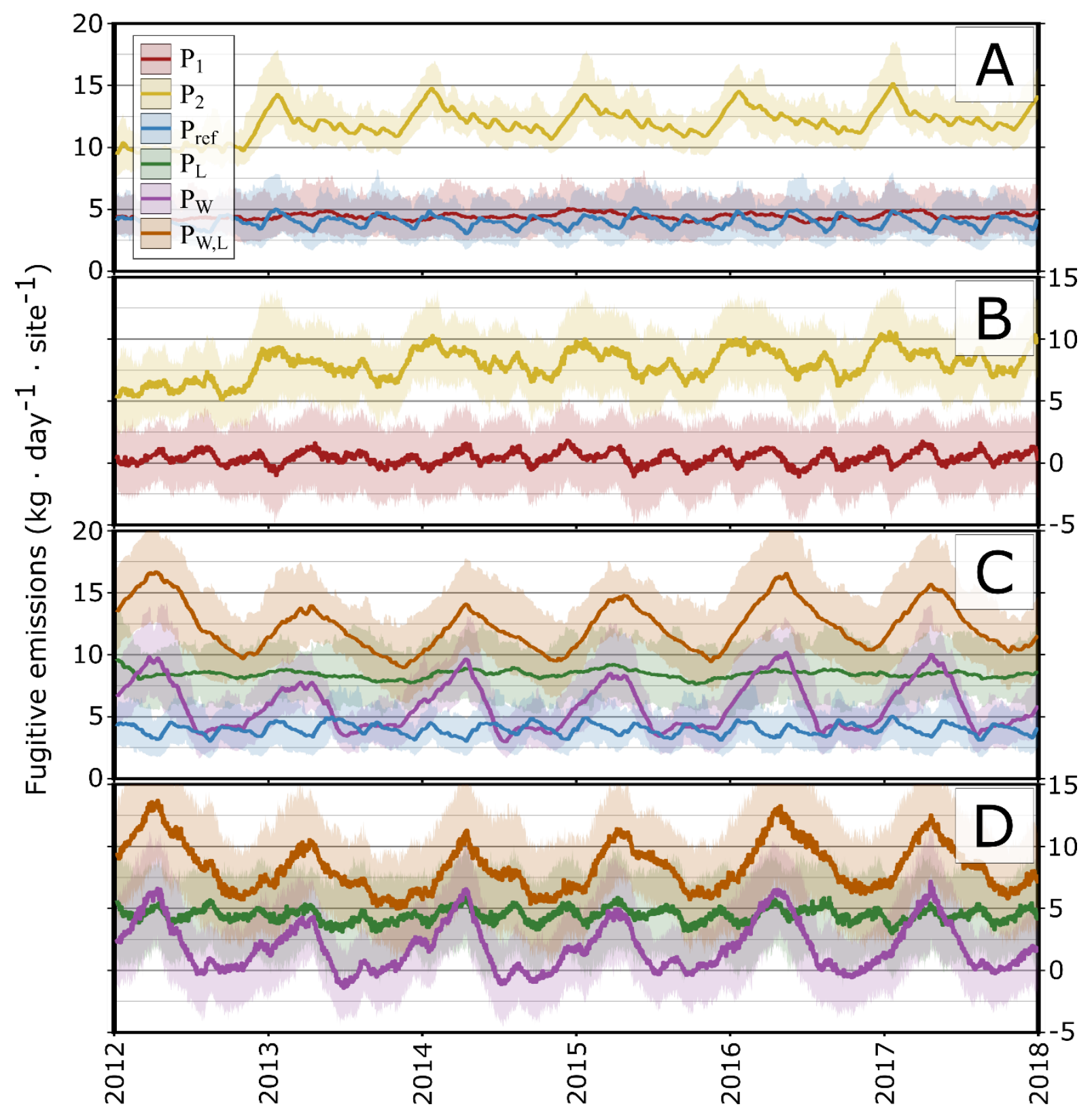

Figure 2. Fugitive emissions observed under each program. Lines show daily emissions averaged across 25 simulations $( \pm 2 \sigma)$. A and $\mathrm{C}$ show average site-level emissions, while $\mathrm{B}$ and $\mathrm{D}$ show candidate programs differenced against the regulatory program $\left(\mathrm{P}_{\mathrm{X}}-\mathrm{P}_{\mathrm{ref}}\right)$. In $\mathrm{B}$ and $\mathrm{D}$, equivalence occurs at $\mathrm{y}=0$. 


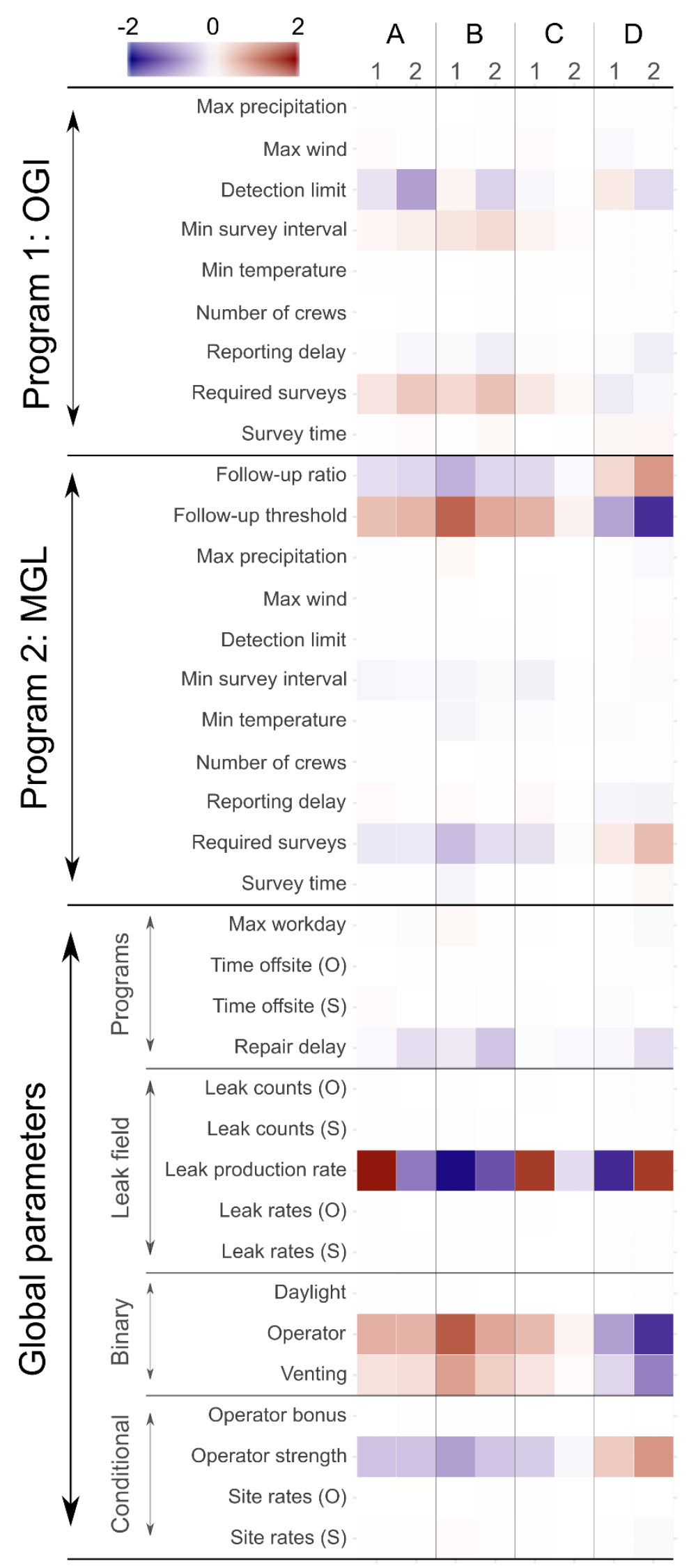


Figure 3. Sensitivity analysis results. Darker colours indicate more sensitive inputs; see S3 for derivation of sensitivity index $S$. Outputs are: (A) median number of active leaks, (B) median number of days leaks remain active before discovered, (C) mean daily site emissions, (D) cumulative number of repaired leaks. For each output, column 1 calculates $S$ using absolute differences between programs ( $M G L-O G I)$, while column 2 uses a ratio $(M G L / O G I)$. Values of $S$ near zero suggest that the input parameter has little influence over the equivalence metric, though don't necessarily indicate that the program is unaffected by the input.
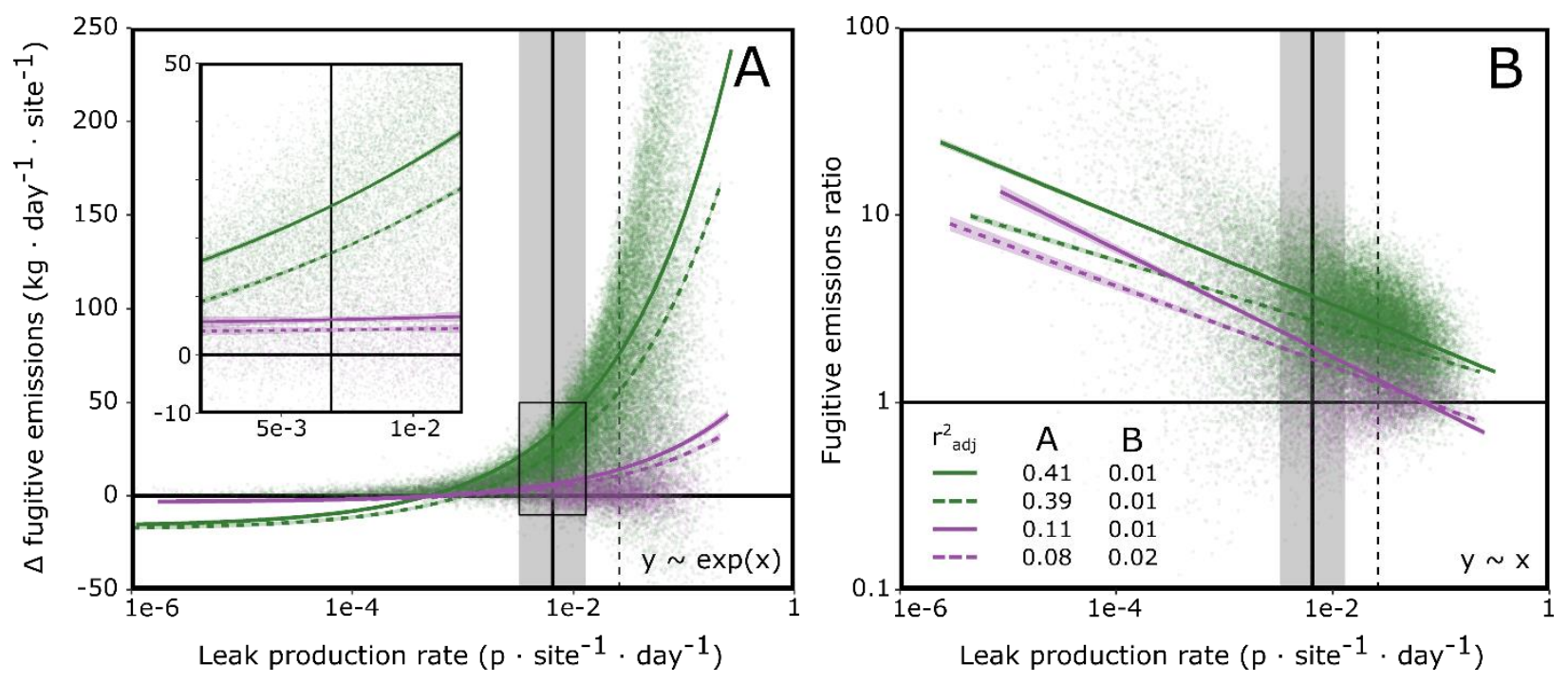

Figure 4. Differences (A; MGL - OGI) and ratios (B; MGL/OGI) of fugitive emissions as a function of LPR. Each dot compares a close-range and a screening program that share global parameters. Purple and green lines show operator presence and absence, respectively (i.e. NRR turned on or off). Dashed lines show presence and solid lines show absence of vented emissions. The dashed vertical line is the mean LPR from the SA sampling distribution (0.026), established using Alberta data. The solid vertical line is the LPR value used in FEAST and the shaded region is the LPR SA range evaluated in Kemp et al. ${ }^{11}$ 


\section{Abbreviations}

\begin{tabular}{|l|l|}
\hline alt-LDAR & Alternative LDAR \\
\hline AVO & Audial, visual, and olfactory \\
\hline ECCC & Environment and Climate Change Canada \\
\hline FEAST & Fugitive emissions abatement simulation testbed \\
\hline LDAR & Leak detection and repair \\
\hline LPR & Leak production rate \\
\hline MGL & Mobile ground laboratory \\
\hline NRR & Null repair rate \\
\hline OGI & Optical gas imaging \\
\hline reg-LDAR & Regulatory LDAR \\
\hline SA & Sensitivity analysis \\
\hline US & United States \\
\hline
\end{tabular}

\section{Acknowledgements}

We thank GreenPath Energy Ltd., Clearstone Engineering Ltd, the Alberta Energy Regulator, and the Petroleum Technology Alliance of Canada for assistance in curating input datasets. We thank the Natural Sciences and Engineering Research Council of Canada for a Vanier scholarship to TAF and Alberta Innovates for a doctoral scholarship to TAF.

\section{Author contributions}

TAF, MG, TEB, and CHH contributed to project conception and design. TAF, MG, and TEB designed and wrote the model code. TAF conducted the case study, including parameterization, simulation, and analysis. TAF designed and conducted the sensitivity analysis and drafted the manuscript. All authors contributed to writing, review and approval of the final manuscript.

\section{Declaration of interests}

The authors declare no competing interests.

\section{Model code and case study inputs}

\section{https://github.com/tarcadius/LDAR Sim/tree/Fox etal 2020 preprint}

\section{References}

(1) Government of Canada, E. and C. C. C. Environment and Climate Change Canada - Climate Change - National Inventory Report 1990-2014: Greenhouse Gas Sources and Sinks in Canada Executive Summary https://www.ec.gc.ca/ges-ghg/default.asp?lang=En\&n=662F9C56-1 (accessed Jul 5, 2017).

(2) EPA. Inventory of U.S. Greenhouse Gas Emissions and Sinks, 1990-2017; US Environmental Protection Agency, 2019.

(3) Alberta Energy Regulator. Directive 060: Upstream Petroleum Industry Flaring, Incinerating, and Venting; 2018; p 99.

(4) Government of Canada. Regulations Respecting Reduction in the Release of Methane and Certain Volatile Organic Compounds (Upstream Oil and Gas Sector) (SOR/2018-66). 2018. 
(5) Schwietzke, S.; Harrison, M.; Lauderdale, T.; Branson, K.; Conley, S.; George, F. C.; Jordan, D.; Jersey, G. R.; Zhang, C.; Mairs, H. L. Aerially-Guided Leak Detection and Repair: A Pilot Field Study for Evaluating the Potential of Methane Emission Detection and Cost-Effectiveness. J. Air Waste Manag. Assoc. 2018, No. just-accepted.

(6) Fox, T. A.; Barchyn, T. E.; Risk, D.; Ravikumar, A. P.; Hugenholtz, C. H. A Review of CloseRange and Screening Technologies for Mitigating Fugitive Methane Emissions in Upstream Oil and Gas. Environ. Res. Lett. 2019, 14 (5), 053002.

(7) CDPHE. Alternative AIMM Guidance \& Procedures. May 2018.

(8) Fox, T. A.; Ravikumar, A. P.; Hugenholtz, C. H.; Zimmerle, D.; Barchyn, T. E.; Johnson, M.; Lyon, D.; Taylor, T. A Methane Emissions Reduction Equivalence Framework for Alternative Leak Detection and Repair Programs. Elementa 2019, 7 (1).

(9) Brandt, A. R.; Heath, G. A.; Cooley, D. Methane Leaks from Natural Gas Systems Follow Extreme Distributions. Environ. Sci. Technol. 2016, 50 (22), 12512-12520.

(10) Ravikumar, A. P.; Sreedhara, S.; Wang, J.; Englander, J.; Roda-Stuart, D.; Bell, C.; Zimmerle, D.; Lyon, D.; Mogstad, I.; Ratner, B. Single-Blind Inter-Comparison of Methane Detection Technologies-Results from the Stanford/EDF Mobile Monitoring Challenge. Elem Sci Anth 2019, $7(1)$.

(11) Kemp, C. E.; Ravikumar, A. P.; Brandt, A. R. Comparing Natural Gas Leakage Detection Technologies Using an Open-Source Virtual Gas Field Simulator. Environ. Sci. Technol. 2016, 50 (8), 4546-4553. https://doi.org/10.1021/acs.est.5b06068.

(12) Peckham, S. D.; Hutton, E. W.; Norris, B. A Component-Based Approach to Integrated Modeling in the Geosciences: The Design of CSDMS. Comput. Geosci. 2013, 53, 3-12.

(13) Dee, D. P.; Uppala, S. M.; Simmons, A. J.; Berrisford, P.; Poli, P.; Kobayashi, S.; Andrae, U.; Balmaseda, M. A.; Balsamo, G.; Bauer, P.; et al. The ERA-Interim Reanalysis: Configuration and Performance of the Data Assimilation System. Q. J. R. Meteorol. Soc. 2011, 137 (656), 553-597. https://doi.org/10.1002/qj.828.

(14) Ravikumar, A. P.; Brandt, A. R. Designing Better Methane Mitigation Policies: The Challenge of Distributed Small Sources in the Natural Gas Sector. Environ. Res. Lett. 2017, 12 (4), 044023. https://doi.org/10.1088/1748-9326/aa6791.

(15) Allen, D. T.; Torres, V. M.; Thomas, J.; Sullivan, D. W.; Harrison, M.; Hendler, A.; Herndon, S. C.; Kolb, C. E.; Fraser, M. P.; Hill, A. D.; et al. Measurements of Methane Emissions at Natural Gas Production Sites in the United States. Proc. Natl. Acad. Sci. 2013, 110 (44), 17768-17773.

(16) Johnson, M. R.; Tyner, D. R.; Conley, S.; Schwietzke, S.; Zavala-Araiza, D. Comparisons of Airborne Measurements and Inventory Estimates of Methane Emissions in the Alberta Upstream Oil and Gas Sector. Environ. Sci. Technol. 2017.

(17) Brandt, A. R.; Heath, G. A.; Kort, E. A.; O’Sullivan, F.; Pétron, G.; Jordaan, S. M.; Tans, P.; Wilcox, J.; Gopstein, A. M.; Arent, D.; et al. Methane Leaks from North American Natural Gas Systems. Science 2014, 343 (6172), 733-735.

(18) Zavala-Araiza, D.; Lyon, D. R.; Alvarez, R. A.; Davis, K. J.; Harriss, R.; Herndon, S. C.; Karion, A.; Kort, E. A.; Lamb, B. K.; Lan, X.; et al. Reconciling Divergent Estimates of Oil and Gas Methane Emissions. Proc. Natl. Acad. Sci. 2015, 112 (51), 15597-15602.

(19) Vaughn, T. L.; Bell, C. S.; Pickering, C. K.; Schwietzke, S.; Heath, G. A.; Pétron, G.; Zimmerle, D. J.; Schnell, R. C.; Nummedal, D. Temporal Variability Largely Explains Top-down/Bottom-up Difference in Methane Emission Estimates from a Natural Gas Production Region. Proc. Natl. Acad. Sci. 2018, 201805687.

(20) Johnson, D.; Heltzel, R.; Oliver, D. Temporal Variations in Methane Emissions from an Unconventional Well Site. ACS Omega 2019, 4 (2), 3708-3715.

(21) Rhodes, B. C. PyEphem: Astronomical Ephemeris for Python. Astrophys. Source Code Libr. 2011.

(22) CAPP. Management of Fugitive Emissions at Upstream Oil and Gas Facilities; Canadian Association of Petroleum Producers, 2007; p 59. 


\section{Supplementary Information}

\section{An agent-based model for estimating emissions reduction equivalence among leak detection and repair programs}

Thomas A. Fox ${ }^{*}$, Mozhou Gao, Thomas E. Barchyn, Yorwearth L. Jamin ${ }^{\ddagger}$, Chris H. Hugenholtz

${ }^{\dagger}$ Centre for Smart Emissions Sensing Technologies, University of Calgary, 2500 University Drive NW, Calgary, AB, T2N 1N4, Canada

† Clearstone Engineering Ltd., 9006 Ave SW \#700, Calgary, AB, T2P 3K2, Canada

*Correspondence to: thomas.fox@ucalgary.ca 


\section{CONTENTS}

$\begin{array}{ll}\text { S1. OPERATOR DETECTION S3 } & \text { SPNON }\end{array}$

$\begin{array}{ll}\text { S2. CASE STUDY PARAMETERIZATIONS } & \text { S7 }\end{array}$

$\begin{array}{lr}\text { S3. SENSITIVITY ANALYSIS } & \text { S10 }\end{array}$

$\begin{array}{lr}\text { S4. SUPPORTING TABLES } & \text { S14 }\end{array}$

$\begin{array}{lr}\text { S5 SUPPORTING FIGURES } & \text { S15 }\end{array}$

$\begin{array}{lr}\text { S6. REFERENCES } & \text { S20 }\end{array}$ 


\section{S1. OPERATOR DETECTION}

In the past, LDAR simulation modeling has operated on the assumption that the input emissions data represents a 'pre-LDAR' steady-state baseline, which must ensure that (1) system-total emission rate is in a steady state, (2) system-total number of leaks is in a steady state, and therefore (3) the relative proportion of leak sizes is in a steady state. ${ }^{1,2}$ In the absence of LDAR, two variables define the leak pool. The first is LPR, which increases the number of leaks. The second, opposing process is the Null Repair Rate (NRR), which removes leaks to place an upper bound on the size of the leak pool. The NRR represents periodic inspection, maintenance, and repair by operators outside of an LDAR program. Together, LPR and NRR work in opposition to maintain the equilibrated baseline in the absence of LDAR.

To maintain a steady state, leak removal must equal production over time, both in terms of counts and total emissions. In LDAR-Sim, an 'operator' agent is used for null repair. The operator module is optional and can work both in the presence and absence of an LDAR program. At a specified interval (typically on Mondays), an operator visits each site (i.e. facility) in the simulation. At each site, each leak has a detection probability $(N R R)$ at time $t$ of:

$$
N R R=\frac{L P R \cdot \mathrm{V}}{\overline{L_{t 0}}} \cdot \frac{L_{t}}{L_{t 0}}+\frac{p_{\max } \cdot Q_{i}}{Q_{\max }}
$$

where $V$ is the visitation interval in days (in this case, 7 ), $L_{t 0}$ is the global leak count at initialization, $L_{t}$ is the number of active leaks at time $\mathrm{t}$, and $\overline{L_{t 0}}$ is the mean number of leaks per site at initialization. Note that units differ for $\operatorname{NRR}\left(p \cdot l e a k^{-1} \cdot d a y^{-1}\right)$, which is applied to individual leaks, and LPR $\left(p \cdot s i t e^{-1} \cdot d a y^{-1}\right)$, which is applied at the site level. We therefore 
scale LPR to the leak level using $\overline{L_{t 0}}$. The unitless term $\frac{L_{t}}{L_{t 0}}$ is used to maintain the steady state, adjusting NRR in proportion to the number of active leaks relative to initialization.

The final term is used in SA to explore the possibility that larger leaks are more easily identified and readily repaired by facility operators. In FEAST, LPR is applied to update the Markov state of non-leaking components. These components can be thought of as leak 'slots' that get filled with random draws from the leak-size distribution and then emptied via null repair or LDAR. If a big leak is drawn, the leak will probably be detected during the next LDAR survey and removed from the slot. However, if the leak is very small and unlikely to be detected, it will occupy that component. As time goes on, a greater proportion of the leak slots can become filled with undetectable leaks, unless they are randomly removed from the leak pool via the null repair rate. FEAST's Markov chain therefore requires that small leaks be equally likely as large leaks to be repaired in the null scenario. Otherwise, small leaks would grow to dominate the available components, 'taking up space'. However, it is reasonable to assume that larger leaks are preferentially removed from the leak pool during null repair because (1) large leaks are more easily detected by human audial, visual, and olfactory (AVO) senses, and (2) if discovered, large leaks are of greater safety and environmental concern.

Changing the NRR to be a function of leak size is challenging because it can destabilize the equilibrium of the leak pool. If we assume that removal rate is a function of leak size, the production rate must also change to keep the leak-size distribution in equilibrium. In other words, if larger leaks are more likely to be removed, then production of larger leaks must increase to maintain the relative proportion of large leaks, and production of small leaks must decrease to maintain the relative proportion of small leaks. Ideally, LPR and NRR should be derived independently. However, measuring these values independently is challenging (if not 
impossible), as NRR is nearly always implicitly measured at the time LPR is measured. One solution is to measure NRR and subtract it from LPR. However, measuring NRR may not be as simple as asking operators to keep track of each leak they actively repair; leaks may resolve themselves through changes in pressure and production, the emergence of different leaks nearby, or unintentionally during maintenance or changes in equipment. Furthermore, LPR and NRR likely vary by producer, basin, and facility.

In LDAR-Sim, we adopt a simple method of adjusting the operator detection function. This method ignores LPR-NRR dependence and is used only in the SA for heuristic purposes. A sigmoid-shape curve similar to those used for modeling detection limits for other methods could be used. ${ }^{3}$ However, parameterizing the model is not currently possible, as there exists no empirical evidence describing AVO detection probability as a function of leak size. Here, $p_{\max }$ (called 'operator bonus' in the SA) is the marginal increase in detection probability assigned to the largest source in the empirical dataset, $Q_{i}$ is the emission rate of the leak under consideration, and $Q_{\max }$ is the largest maximum emission rate in the dataset. In this way, each leak is assigned the same base probability of detection in addition to a 'bonus' chance of detection, which is a function of emission rate. Leaks emitting at the highest possible emission rate, as defined by the empirical leak-size distribution, will receive a bonus detection probability of $p_{\max }$. For the smallest leaks, $\frac{Q_{i}}{Q_{\max }}$ approaches zero, so the adjustment is negligible (Figure S3).

This approach makes two assumptions. First, detection probabilities increase with the size of the leak, up to the user-defined maximum. However, detection probabilities are not similarly or opposingly reduced for small leaks. Although it is reasonable to assume that large leaks will be quickly identified by operators (for an extreme example, recall the Aliso Canyon leak in northern Los Angeles, US during 2015-2016), small leaks may continue to be repaired at the base level 
due to periodic maintenance. Second, this approach assumes that LPR remains constant, meaning the baseline will necessarily disequilibriate as 1) large leaks are preferentially detected and repaired, and 2) more leaks are detected in total. Table S3 provides a simple demonstration that making NRR a function of leak size can have a large effect on mitigation, even for small $p_{\max }$ values. 


\section{S2. CASE STUDY PARAMETERIZATIONS}

All empirical inputs used are specific to Alberta. Leak-rate and leak-count distributions come from 2017 data targeting 333 upstream O\&G sites using OGI cameras. ${ }^{4}$ The Clearstone study was broadly representative of Alberta's O\&G sector, involving 63 companies producing natural gas and light, medium, and heavy crude oil. Site-level measurements used to derive empirical vents were acquired using a combination of tracer flux and Gaussian dispersion methods. ${ }^{5}$

We estimate LPR using publicly available LDAR data compiled from nine producers in Alberta between July 2007 and October $2016 .^{6}$ In total, there were 8097 leaks measured, mostly at larger facilities such as gas plants and multi-well batteries. Of 1283 unique site visits, 436 were documented repeat visits of a minimum 30-day survey interval. We estimated LPR by dividing leak count by the number of days since the most recent survey. We removed one extreme outlier in which 78 leaks were detected after only 59 days. The distribution of site-level LPR estimates from the GreenPath study is shown in Figure S4 (note log scale). In FEAST, a constant LPR of 1e-5 per component is used. As FEAST assumes 650 components per site, the site-level LPR is $\sim 0.0065$ (vertical red line in Figure S4). Differences between FEAST and our estimate could be due to infrastructure types, region, methodological discrepancies in data acquisition, and validity of assumptions. Specifically, both studies assume that (1) leaks reported during the first survey were repaired, and (2) NRR is inconsequential. We cannot comment on the data used to estimate LPR in FEAST, but the data used in this study are highly unlikely to meet the assumption of complete repair. Repair rates for the dataset may be as low as $8 \%$, due to incomplete record keeping and flexibility to defer repairs at the time of the study, which could lead to a significant overestimation of LPR. ${ }^{6}$ Nevertheless, LPR values estimated in this study span over two orders of magnitude. In FEAST, LPR was the most sensitive parameter, despite using only a limited 
range in the SA (the shaded region in Figure S4). For this demonstration we adopt the same LPR as FEAST (0.0065) but note that both the methods used to estimate LPR here and those in FEAST have several problematic assumptions. Given that NRR is implicitly measured in our LPR estimate, NRR functionality is disabled for this demonstration.

The case study evaluates equivalence of two alt-LDAR programs $\left(\mathrm{P}_{1}\right.$ and $\left.\mathrm{P}_{2}\right)$ against a reference reg-LDAR program $\left(\mathrm{P}_{\mathrm{Ref}}\right)$ configured according to ECCC regulations ${ }^{7}$. Three additional programs $\left(\mathrm{P}_{\mathrm{W}}, \mathrm{P}_{\mathrm{L}}, \mathrm{P}_{\mathrm{W}, \mathrm{L}}\right)$ modify $\mathrm{P}_{\text {Ref }}$ to illustrate the importance of environmental envelopes and labour constraints (Table 1, main text). The $\mathrm{P}_{\mathrm{W}}$ scenario includes an OGI company that limits work in inclement conditions but hires more crews. The $\mathrm{P}_{\mathrm{L}}$ scenario represents a company that will operate in a much broader environmental envelope. The $\mathrm{P}_{\mathrm{W}, \mathrm{L}}$ scenario combines $\mathrm{P}_{\mathrm{W}}$ and $\mathrm{P}_{\mathrm{L}}$. The ECCC $\mathrm{P}_{\text {Ref }}$ does not specify environmental envelopes; we assume that most service providers will work in all but the most extreme conditions: temperatures $>-30{ }^{\circ} \mathrm{C}$ and winds $<$ $20 \mathrm{~m} \cdot \mathrm{s}^{-1}$. In $\mathrm{P}_{\mathrm{W}}$ and $\mathrm{P}_{\mathrm{W}, \mathrm{L}}$ agents are only deployed under preferred weather conditions: $>0{ }^{\circ} \mathrm{C},<$ $5 \mathrm{~m} \cdot \mathrm{s}^{-1}$ wind, and $<10 \mathrm{~mm}$ daily precipitation rate). These thresholds are based loosely on detection modeled performances but are not grounded in legislation or best practices. $^{2}$ Unfortunately, insufficient empirical data exist to inform OGI operational envelopes. Labour constraints are imposed on $\mathrm{P}_{\mathrm{L}}$ and $\mathrm{P}_{\mathrm{W}, \mathrm{L}}$ (one OGI crew instead of three). Neither of the screening programs $\left(\mathrm{P}_{1}\right.$ and $\left.\mathrm{P}_{2}\right)$ are constrained by weather or labour. Both $\mathrm{P}_{1}$ and $\mathrm{P}_{2}$ use the $\mathrm{P}_{\mathrm{Ref}} \mathrm{OGI}$ method configuration for follow-up. Although more complex programs comprising multiple methods are possible, this demonstration focuses on simple programs.

Close-range methods (used in $\mathrm{P}_{\mathrm{Ref}}, \mathrm{P}_{\mathrm{W}}, \mathrm{P}_{\mathrm{L}}, \mathrm{P}_{\mathrm{W}, \mathrm{L}}$, and follow-up) use sigmoidal detection probability curves developed empirically in Ravikumar et al. (2018). For each leak surveyed, shape parameters are sampled from normal distributions $k \sim \mathcal{N}(4.9,0.3)$ and $x_{0} \sim$ 
$\mathcal{N}(0.47,0.01)$, assuming an imaging distance of $3 \mathrm{~m}$. We use single-value detection limits for $\mathrm{P}_{1}$ and $\mathrm{P}_{2}\left(100\right.$ and $\left.5000 \mathrm{~g} \cdot \mathrm{h}^{-1}\right)$, as empirical detection probability curves have not been published for MGLs or aircraft. These values fall within typical ranges reviewed. ${ }^{8}$ In $\mathrm{P}_{1}$ and $\mathrm{P}_{2}$, follow-up crews visit $80 \%$ and $50 \%$ of flagged sites, respectively. Note that both alt-LDAR programs are based on hypothetical technologies and work practices and should not be considered representative of typical MGL or aircraft-based programs. Our aversion to using existing systems for this case study is intentional to avoid promoting any one solution provider. Further, most screening technologies have evolving skills and poorly defined work practices.

A constant survey time of 120 minutes per site is used for OGI surveys, following literature. ${ }^{8}$ The per-site times used for $\mathrm{P}_{1}$ and $\mathrm{P}_{2}$ are heuristics and not representative of any real system or work practice. After each visit, a 'time offsite' increment is added to the workday, which is broadly representative of all driving or flying, breaks, acquiring permits, and unforeseen circumstances. For methods that must travel overland, we do not explicitly account for betweensite distances, which would require modeling of complex and often subjective site selection procedures. Instead, we sample from an empirical distribution of between-site durations acquired during field campaigns in Alberta (unpublished University of Calgary data; available in SI). In the absence of empirical data, $\mathrm{P}_{2}$ uses a constant 10-minute between-site interval. 


\section{S3. SENSITIVITY ANALYSIS}

We conducted 50000 Monte Carlo simulations, each spanning 1095 days plus a 500-day spin-up period. In each run, programs share a set of global parameters (e.g. LPR, repair delays) sampled from broadly representative probability distributions. Method-specific parameters (e.g. detection limits, survey frequency) are sampled independently. The following index $(S)$ is reported:

$$
S=\frac{\left(O_{\text {high }}-O_{\text {low }}\right)}{O_{\sigma}}
$$

The terms used to calculate $S$ depend on whether the SA evaluates a single program (e.g. Figure S5) or the comparison between two programs (Figure 3, main text). In both cases, all parameters are randomly sampled for each SA simulation in order to account for cross-variable sensitivity and possible interaction effects. Given that each parameter is evaluated across a broad range of possible scenarios, many of the SA realizations represent unlikely combinations of input parameters, leading to high output variability that can be used to identify sensitive parameters. Here, variability in outputs that affect equivalence determinations are of greatest interest. However, we begin with the definition of $S$ for a single program, which is simpler: $O_{\text {high }}$ and $O_{\text {low }}$ are the median outputs corresponding to the upper and lower deciles (highest and lowest $10 \%$ ) of the distribution of input samples. The output standard deviation $O_{\sigma}$ standardizes $S$, allowing comparison among different input-output combinations. Put generally, sensitivity of an input parameter is the difference between outputs for the highest inputs and lowest inputs, averaged across all other parameter combinations, and measured in standard deviations of the output distribution. If the difference in outputs is high, so is $S$, and the input variable has a strong effect. 
Calculating $S$ for a SA in which two programs are compared is more complicated. We calculate $O_{\text {high }}$ and $O_{\text {low }}$ first as the difference, and then as the ratio of outputs between two distinct programs sharing the same global parameters. Thus, $O$ should approach zero when programs are equivalent, and $S$ becomes a difference (or ratio) of equivalence measured in standard deviations of the equivalence metric. When $S$ is near zero, the equivalence metric (i.e. difference or ratio of outputs) is independent of the input value.

Two exceptions exist. For binary inputs (e.g. whether venting is considered), median outputs are taken for True/False categories, as deciles are not applicable. When inputs are conditional (e.g. site-level emissions estimates), only inputs that meet the associated condition are considered (e.g. venting is True). Users of LDAR-Sim should note that the SA performed here is specific to a unique set of empirical inputs, environmental conditions, and hypothetical detection methods. We recommend performing a SA each time a new program is evaluated.

Input distributions used for the sensitivity analysis (SA) are shown in Figure S6. All input parameters are listed below. Variables with the suffix '_outliers' or '_samples' are used to permute empirical distributions. These distributions include the leak-size distribution (LSD), leak-count distribution (LCD), site-level emission rate distribution (site_rate), and offsite driving times (offsite_times). For each distribution, a number of outliers (integers typically between 0 and 3) is sampled and either added to or removed from the distribution. For example, if -2 was sampled, the two largest elements in the empirical input distribution would be removed. When a new outlier is added, the value is chosen by doubling the largest element in the distribution. For example, if 2 was sampled, two new outliers would be added to the distribution. The second outlier added would be four times the size of the largest element in the original distribution. Input distributions are then sampled from a truncated normal distribution with a mean equal to 
the length of the original distribution $(l)$ and a standard deviation of one quarter of this value. A lower limit of 10 samples is enforced to ensure proper functioning of the model.

Leak production rate (LPR) is parameterized by fitting a gamma distribution to the empirical distribution of LPR values. For the detection limits listed below, it should be noted that the OGI parameter estimated is variable $\mathrm{x}_{0}$ described in Ravikumar et al., ${ }^{3}$ while the MGL detection limit is an absolute minimum measured in $\mathrm{g} \mathrm{h}^{-1}$. For the variables that require integers, samples are rounded as necessary.

$$
\begin{gathered}
\text { LSD outliers } \sim \operatorname{Normal}(0,1) \\
\text { LSD samples } \sim \operatorname{Normal}(l, l / 4) \\
\text { LCD outliers } \sim \operatorname{Normal}(0,1) \\
\text { LCD samples } \sim \operatorname{Normal}(l, l / 4) \\
\text { Site rate outliers } \sim \operatorname{Normal}(0,1) \\
\text { Site rate samples } \sim \operatorname{Normal}(l, l / 4) \\
\text { Offsite times outliers } \sim \operatorname{Normal}(0,1) \\
\text { Offsite times samples } \sim \operatorname{Normal}(l, l / 4) \\
\text { LPR } \sim \text { Gamma }(0.8328,0.03139) \\
\text { Repair delay } \sim \text { Uniform }(0,100) \\
\text { Maximum work day } \sim \operatorname{Uniform}(6,14) \\
\text { Oponsider daylight } \sim \operatorname{Binomial}(1,0.5) \\
\text { Oponsider operator } \sim \operatorname{Binomial}(1,0.2) \\
\text { Operator bonus } \sim \text { Exponential }(0.2) \\
\text { Oinomial }(1,0.5) \\
\text { Exponential }(0.1)
\end{gathered}
$$


OGI number of crews $\sim$ Poisson $(0.5)+1$

OGI minimum temperature $\sim \operatorname{Normal}(-20,10)$

OGI maximum wind $\sim \operatorname{Normal}(15,3)$

OGI maximum precipitation $\sim$ Uniform $(0,0.1)$

OGI reporting delay $\sim$ Uniform $(0,30)$

OGI time per site $\sim$ Uniform $(30,500)$

OGI required surveys $\sim$ Uniform $(1,4)$

OGI minimum survey interval $\sim \operatorname{Uniform}(0,90)$

OGI detection limit $\sim$ Uniform $(0,2)$

MGL number of crews $\sim$ Poisson $(0.5)+1$

MGL minimum temperature $\sim \operatorname{Normal}(-20,10)$

MGL maximum wind $\sim \operatorname{Normal}(15,3)$

MGL maximum precipitation $\sim$ Uniform $(0,0.1)$

MGL reporting delay $\sim$ Uniform $(0,30)$

MGL time per site $\sim$ Uniform $(1,30)$

MGL required surveys $\sim$ Uniform $(1,4)$

MGL minimum survey interval $\sim \operatorname{Uniform}(0,90)$

MGL detection limit Uniform $(1,100)$

MGL followup threshold $\sim$ Uniform $(0,500)$

MGL followup ratio $\sim \operatorname{Uniform}(0.1,1)$ 


\section{S4. SUPPORTING TABLES}

Table S1. LDAR-Sim output data by day, site, and leak.

\begin{tabular}{|c|c|c|}
\hline By day & By site & By leak \\
\hline date & identifying information* & identifying information* \\
\hline emissions & emissions & emission rate \\
\hline new leak count & initial leak count & date began \\
\hline active leak count & active leak count & date found \\
\hline repaired leak count & repaired leak count & date repaired \\
\hline tag count & flag status & days active \\
\hline proportion sites available ${ }^{\dagger}$ & flag date & repair delay \\
\hline $\operatorname{cost}^{\dagger}$ & flag company & found by (company) \\
\hline redundant tags ${ }^{\ddagger}$ & missed leaks $^{\dagger}$ & found by (crew) \\
\hline effective flags $s^{\S}$ & surveys conducted $^{\dagger}$ & status \\
\hline redundant flags $1^{\S}$ & days since last survey $^{\dagger}$ & \\
\hline \multicolumn{3}{|l|}{ redundant flags $2^{\S}$} \\
\hline \multicolumn{3}{|l|}{ redundant flags $3^{\S}$} \\
\hline \multicolumn{3}{|c|}{$\begin{array}{l}\text { * Each site and leak are assigned a unique identifier. Site name, operator, and location are } \\
\text { output if provided by the user in the input file. }\end{array}$} \\
\hline \multicolumn{3}{|c|}{${ }^{\dagger}$ Reported by method } \\
\hline \multicolumn{3}{|c|}{${ }^{*}$ Reported only for close-range methods and operator } \\
\hline \multicolumn{3}{|c|}{${ }^{\S}$ Reported only for screening methods } \\
\hline \multicolumn{3}{|c|}{ " Can be active, repaired, or tagged } \\
\hline
\end{tabular}

Table S2. Case study results.

\begin{tabular}{lrrrrrr} 
& $\mathrm{P}_{\text {Ref }}$ & $\mathrm{P}_{\mathrm{W}}$ & $\mathrm{P}_{\mathrm{L}}$ & $\mathrm{P}_{\mathrm{W}, \mathrm{L}}$ & $\mathrm{P}_{1}$ & $\mathrm{P}_{2}$ \\
\hline Method & OGI & OGI & OGI & OGI & Truck & Aircraft \\
Proportion sites available & $1.0 \pm 0.1$ & $0.4 \pm 0.4$ & $1.0 \pm 0.1$ & $0.4 \pm 0.4$ & $1.0 \pm 0.1$ & $1.0 \pm 0.1$ \\
Sites flagged per day & - & - & - & - & $7.3 \pm 1.2$ & $2.6 \pm 2.6$ \\
Leaks tagged per day & $7.5 \pm 4.9$ & $7.6 \pm 8.2$ & $7.6 \pm 3.0$ & $7.6 \pm 6.4$ & $7.5 \pm 3.0$ & $7.0 \pm 7.4$ \\
${\text { Emissions }\left(\mathrm{kg} \cdot \text { site }^{-1} \cdot \text { day }^{-1}\right)}$ 4.0 & 4.2 & $5.9 \pm 2.3$ & $8.4 \pm 1.5$ & $12.3 \pm 2.7$ & $4.4 \pm 1.1$ & $11.9 \pm 1.6$ \\
\hline
\end{tabular}

Table S3. Approximate number of active leaks and daily emissions for 500 sites under a range of $p_{\max }$ values (simulations conducted without LDAR). Note that if the largest leaks have only a 5\% chance of being detected by operators during weekly visits (gold line in Figure S3), system-wide emissions fall by $>50 \%$.

\begin{tabular}{rcccccc} 
& $\mathbf{0 . 0 0}$ & $\mathbf{0 . 0 5}$ & $\mathbf{0 . 1 0}$ & $\mathbf{0 . 1 5}$ & $\mathbf{0 . 2 0}$ & $\mathbf{0 . 2 5}$ \\
\cline { 2 - 7 } Active leaks & 3400 & 3100 & 2800 & 2500 & 2400 & 2400 \\
Emissions (kg) & 49000 & 20000 & 13000 & 9000 & 8000 & 7000
\end{tabular}




\section{All flags}

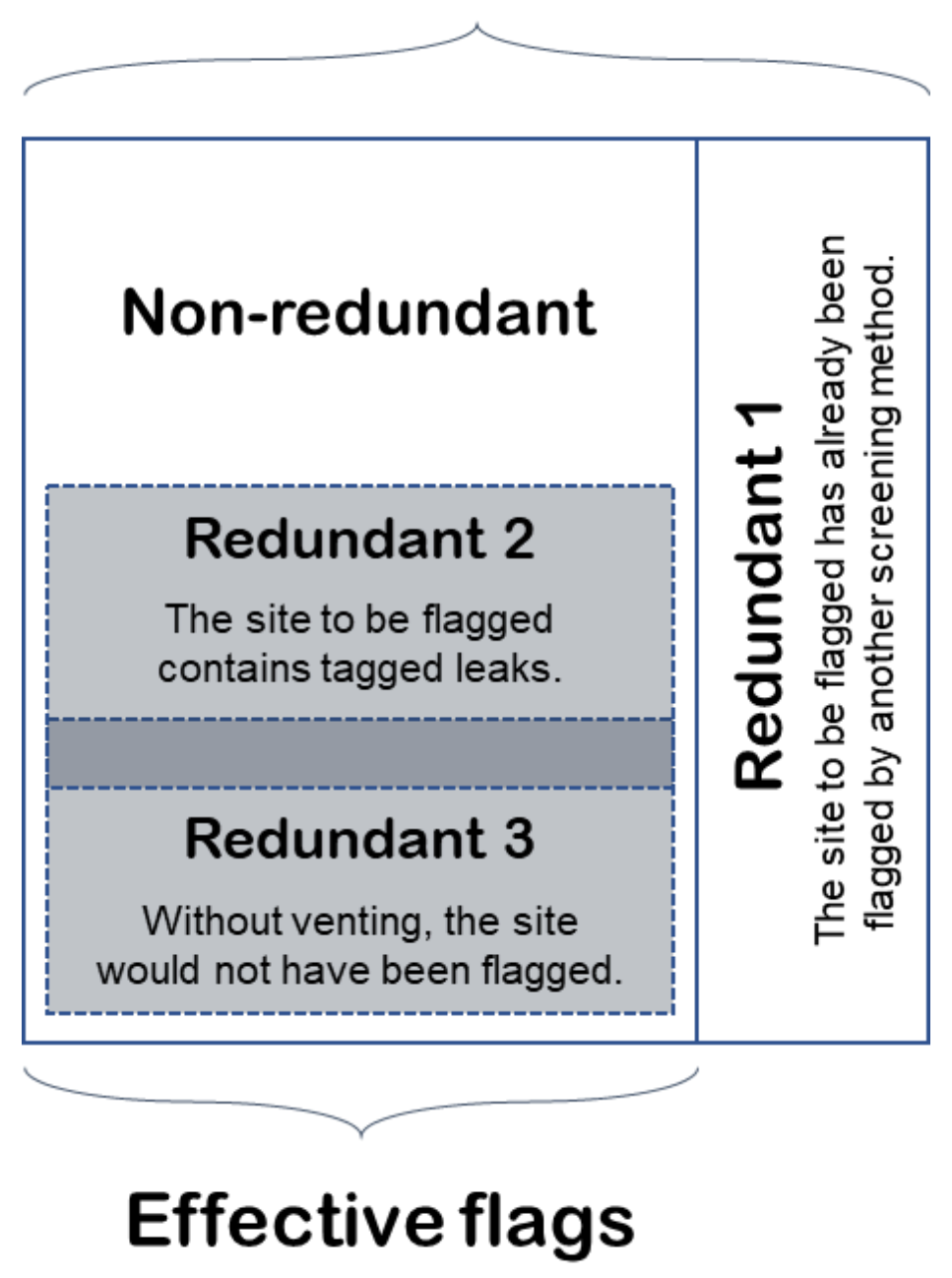

Figure S1. Illustration of the three types of redundancies tracked in LDAR-Sim. 

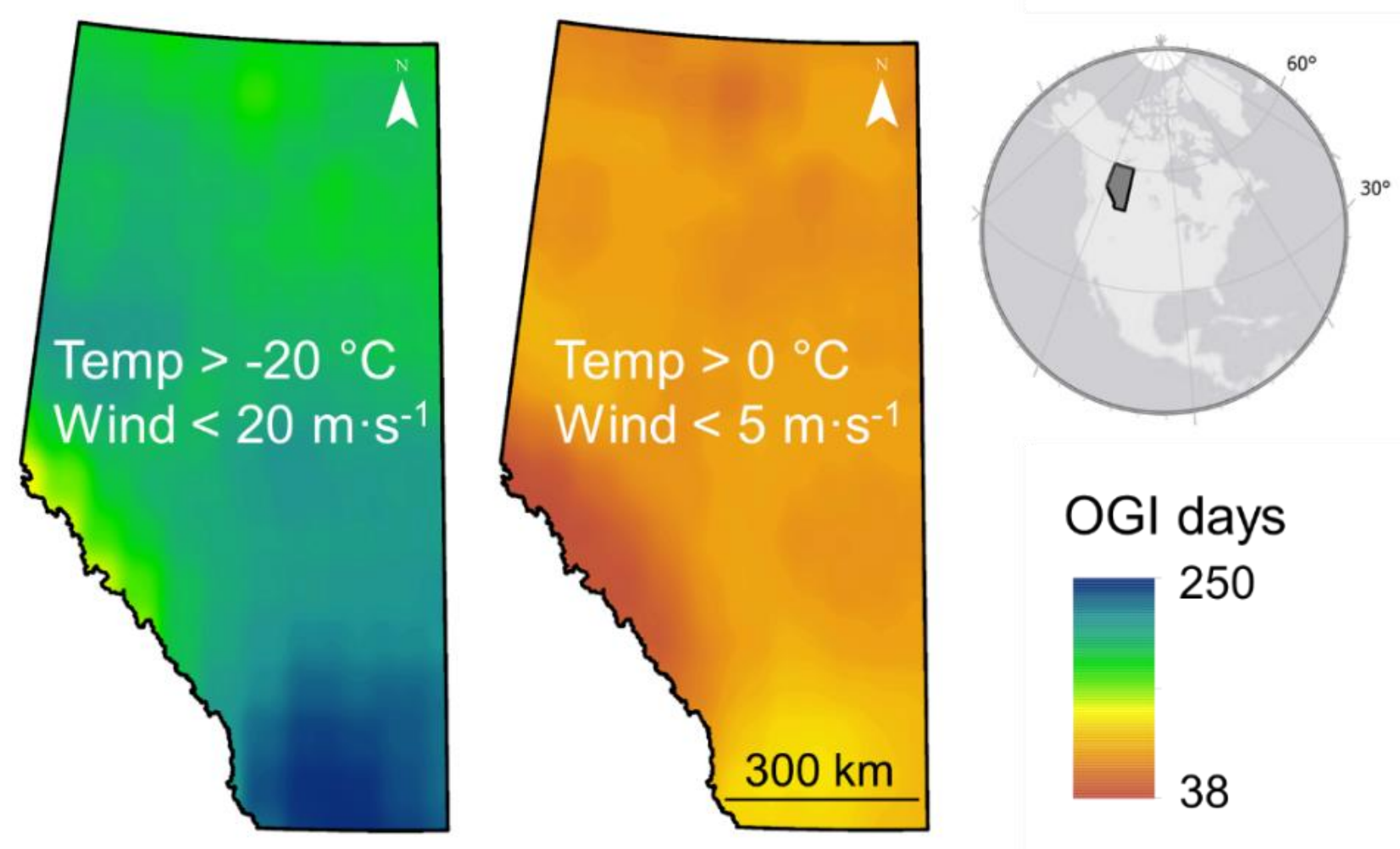

\section{OGI days}

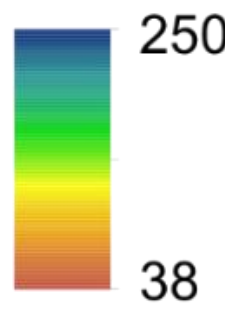

Figure S2. The spatial distribution of OGI suitability in Alberta for different deployment thresholds. On the left, crews are deployed if warmer than $-20{ }^{\circ} \mathrm{C}$ and winds are below $20 \mathrm{~m} \cdot \mathrm{s}^{-1}$. On the right, stricter thresholds are used, resulting in fewer deployment days. 


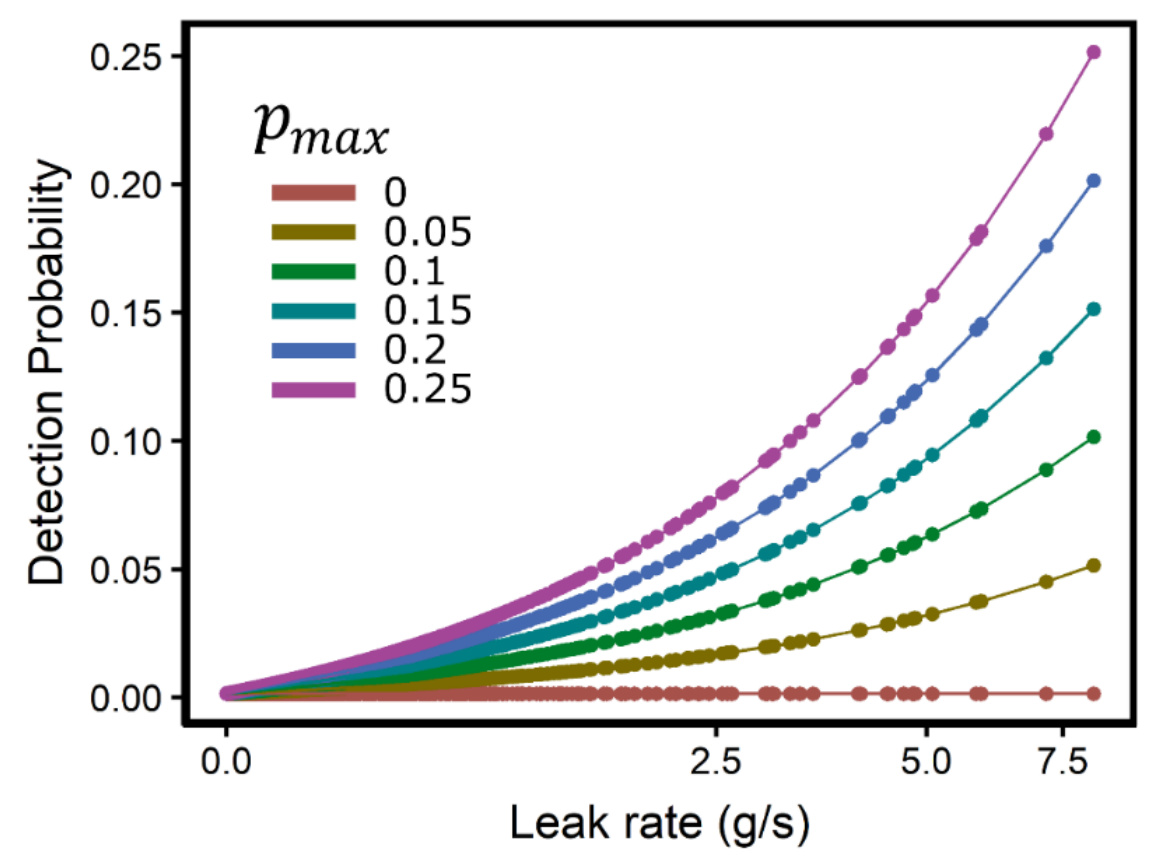

Figure S3. Detection probability curves for six possible $p_{\max }$ values using the Fort Worth Air Quality Study. For example, the gold line $\left(p_{\max }=0.05\right)$ assumes operators have a $5 \%$ chance of detecting the largest leak in the dataset in NRR calculations.

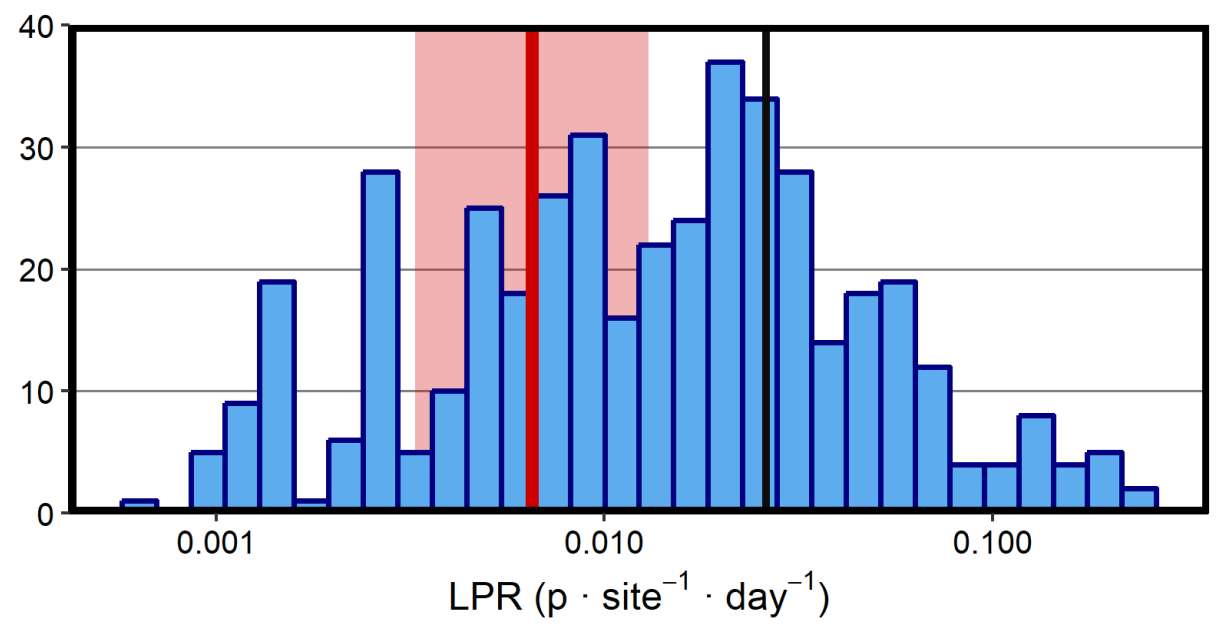

Figure S4. Empirical frequency distribution of leak production rates in Alberta. The mean (vertical black line) is 0.026. The red line is the LPR value used in FEAST and in this LDAR-Sim demonstration. The shaded region is the LPR sensitivity analysis range evaluated in Kemp et al. (2016). 


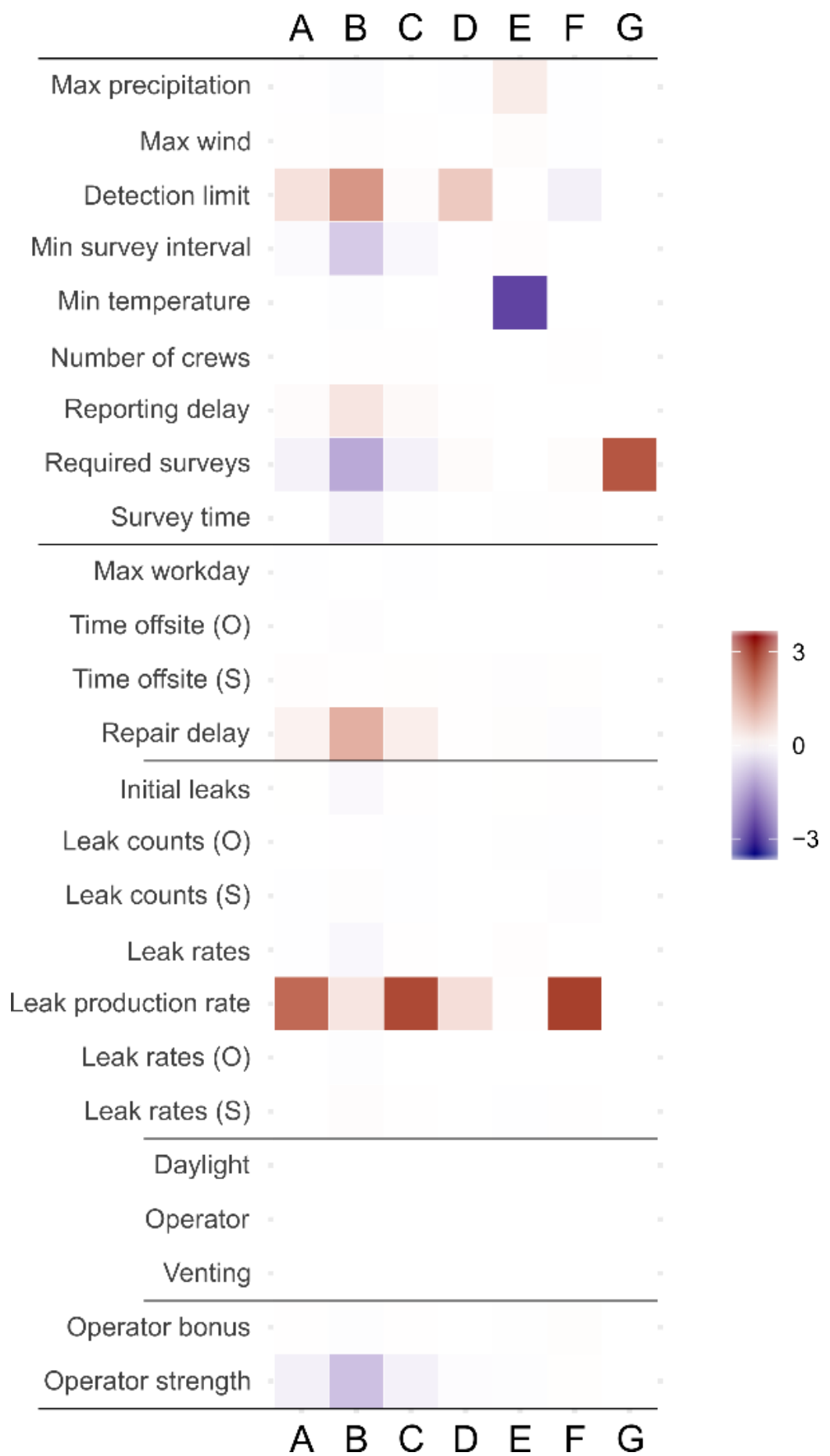

Figure S5. SA results for a single OGI program. Rows are inputs and columns are outputs: (A) number of active leaks, (B) number of days leaks are active, (C) daily emissions, (D) missed leaks, (E) proportion of sites available for LDAR, $(\mathrm{F})$ repaired leaks, and $(\mathrm{G})$ surveys conducted. 

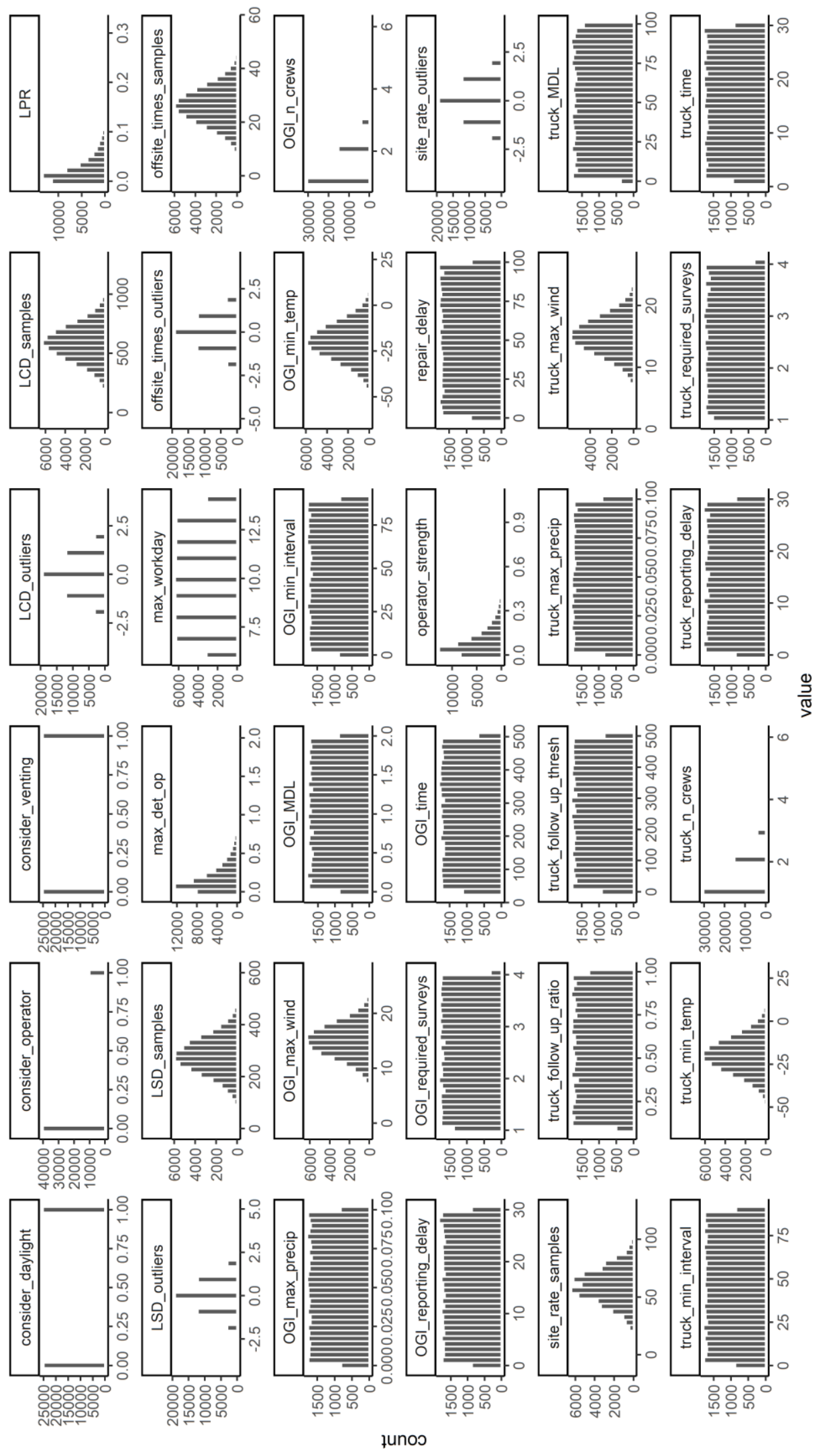

Figure S6. Sampling distributions used in the SA ( $=50$ 000). 


\section{S6. REFERENCES}

(1) Kemp, C. E.; Ravikumar, A. P.; Brandt, A. R. Comparing Natural Gas Leakage Detection Technologies Using an Open-Source Virtual Gas Field Simulator. Environ. Sci. Technol. 2016, 50 (8), 4546-4553. https://doi.org/10.1021/acs.est.5b06068.

(2) Ravikumar, A. P.; Brandt, A. R. Designing Better Methane Mitigation Policies: The Challenge of Distributed Small Sources in the Natural Gas Sector. Environ. Res. Lett. 2017, 12 (4), 044023. https://doi.org/10.1088/1748-9326/aa6791.

(3) Ravikumar, A. P.; Wang, J.; McGuire, M.; Bell, C. S.; Zimmerle, D.; Brandt, A. R. Good versus Good Enough? Empirical Tests of Methane Leak Detection Sensitivity of a Commercial Infrared Camera. Environ. Sci. Technol. 2018.

(4) Clearstone Engineering Ltd. Update of Equipment, Component and Fugitive Emission Factors for Alberta Upstream Oil and Gas.; Technical Report; 2018; p 185.

(5) Zavala-Araiza, D.; Herndon, S. C.; Roscioli, J. R.; Yacovitch, T. I.; Johnson, M. R.; Tyner, D. R.; Omara, M.; Knighton, B. Methane Emissions from Oil and Gas Production Sites in Alberta, Canada. Elem Sci Anth 2018, 6 (1).

(6) GreenPath Energy. Historical Canadian Fugitive Emissions Management Program Assessment; Technical Report; Petroleum Technology Alliance Canada, 2017; p 47.

(7) Government of Canada. Regulations Respecting Reduction in the Release of Methane and Certain Volatile Organic Compounds (Upstream Oil and Gas Sector) (SOR/2018-66). 2018.

(8) Fox, T. A.; Barchyn, T. E.; Risk, D.; Ravikumar, A. P.; Hugenholtz, C. H. A Review of CloseRange and Screening Technologies for Mitigating Fugitive Methane Emissions in Upstream Oil and Gas. Environ. Res. Lett. 2019, 14 (5), 053002. 